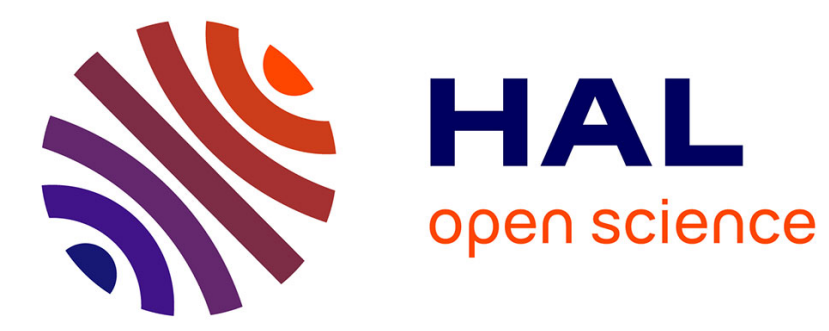

\title{
Appareils et dispositifs divers. Micromanomètre
}

\author{
A. Henry
}

\section{To cite this version:}

A. Henry. Appareils et dispositifs divers. Micromanomètre. J. Phys. Theor. Appl., 1913, 3 (1), pp.652-664. 10.1051/jphystap:019130030065200 . jpa-00242070

\section{HAL Id: jpa-00242070 https://hal.science/jpa-00242070}

Submitted on 1 Jan 1913

HAL is a multi-disciplinary open access archive for the deposit and dissemination of scientific research documents, whether they are published or not. The documents may come from teaching and research institutions in France or abroad, or from public or private research centers.
L'archive ouverte pluridisciplinaire HAL, est destinée au dépôt et à la diffusion de documents scientifiques de niveau recherche, publiés ou non, émanant des établissements d'enseignement et de recherche français ou étrangers, des laboratoires publics ou privés. 


\section{APPAREILS ET DISPOSITIFS DIVERS. MICROMANOMÈTRE ;}

Par M. A. HENRY (1).

Cet appareil est un perfectionnement du manomètre de Kretz.

Deux ampoules cylindriques $A$ et $B$ sont en relation par un tube capillaire, sensiblement horizontal, dont le rayon est d'un millimètre environ ( $f g .1$ et 2 ).

Un même liquide remplit les deux ampoules et le tube capillaire; toutefois la masse liquide est coupée par une bulle de gaz I servant d'index mobile dans le tube capillaire.

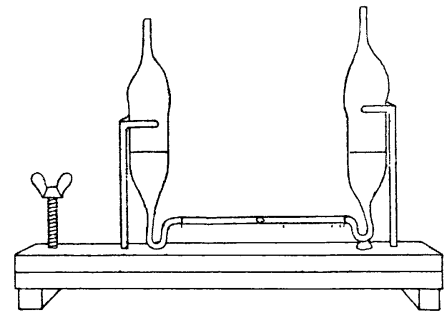

FIG. 1.

L'équilibre étant établi, si l'on accroît la pression du côté A de $x$ baryes, il s'établit entre les niveaux des ampoules une différence $h$. Avec un liquide de densité $d$, on voit immédiatement que la valeur de $x$ est :

$$
x=h d g .
$$

La bulle-index s'est déplacée d'une longueur $l$, telle que le volume décrit par elle soit égal au volume du liquide sorti de A.

En désignant les sections des ampoules et du tube capillaire par $S$ et $s$, on a l'équation :

$$
\frac{\mathrm{S} h}{2}=s l
$$

(1) Cette si remarquable et si ingénieuse étude expérimentale de M. A. Henry, professeur de physique au Lycée de Reims, nous a été remise par M. Carlo Bourlet qui devait en revoir l'épreuve. L'éminent professeur du Conservatoire des Arts et Métiers, enlevé lui aussi, par une mort soudaine, à la science et à l'enseignement, n’a pu réaliser ce projet. Nous publions donc le travail sans modifications. 
Combinant les équations (1) et (2), il vient :

$$
x=2 \frac{s}{\mathrm{~S}} l d g .
$$

Calculant le déplacement $l$ pour une valeur de $x$ équivalant à 1 millimètre d'eau avec $\frac{\mathrm{S}}{\mathrm{s}}=100$, on obtient 50 millimètres (pour $d=1$ ). En augmentant le rapport $\mathrm{S}: s$ on prévoit une grande sensibilité. On pourrait d'ailleurs augmenter séparément la surface libre de l'ampoule B.

Cette prévision est purement théorique : en fait, il faut chercher quel accroissement de pression provoque le démarrage de la bulle; or il se trouve qu'elle obéit à un très faible accroissement de pression, à la condition d'utiliser un liquide convenable. L'eau doit être rejetée, car, en peu de temps, elle provoque en attaquant le verre la formation d'aspérités qui empêchent le glissement.

Des liquides sans action sur le verre s'imposent. Dans le but d'atténuer les déformations aux extrémités de la bulle, on prendra des liquides à faible tension superficielle et, afin d'avoir un glissement assez rapide dans le tube capillaire, on choisira des liquides peu visqueux.

Le tétrachlorure de carbone $(d=1,6)$ et l'acétone $(d=0,8)$ conviennent bien.

L'acétone ordinaire étant altérable à l'air, les recherches ont porté surtout sur le tétrachlorure de carbone.

On a essayé si une goutte de ce liquide tombant dans l'ampoule A suffirait à faire glisser la bulle. Cette dernière obéit immédiatement. Dans les ampoules présentant une section droite quatre cents fois plus grande que celle du tube capillaire, on a laissé tomber des grains de plomb de grosseur de plus en plus faible. Le plus petit de la série soulevant la surface libre de $\frac{1}{1600}$ de millimètre déplaçait encore la bulle-index. Cette hauteur de tétrachlorure correspond à $1 \mu$ d'eau.

En opérant de même dans la seconde ampoule avec un plomb de même grosseur, la bulle glisse encore. On pourrait craindre, à cause des effets de capillarité aux cercles de raccordement, que la surface libre ne participe pas tout entière au relèvement. C'est pourquoi on a cherché autrement la sensibilité. Le micromanomètre est posé sur une longue ligne horizontale dont l'une des extrémités peut se relever 
à l'aide d'une vis micrométrique. On lève la règle de ce côté jusqu'à ce que l'on voie la bulle-index se mouvoir. Par une règle de trois on peut calculer de combien une surface libre a été élevée par rapport à l'autre. On constate encore que la bulle se déplace quand on a fait varier les niveaux de 1:1600 de millimètre environ, ce qui confirme le résultat précédent. Il valait mieux essayer sur l'appareil une vraie force élastique de gaz. Si l'on se rend compte de la faible variation de température d'un gaz enfermé dans l'ampoule capable de provoquer une variation de pression de cet ordre, on voit que la vérification est difficile par cette voie. Cependant, à la condition de laisser

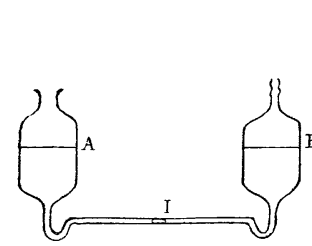

FIG. 2.

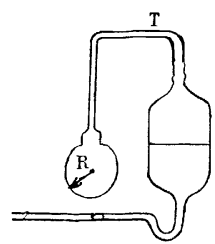

Fig. 3.

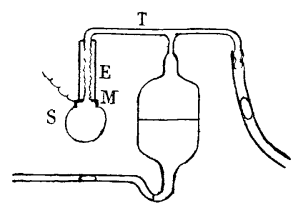

Fig. 4 .

le micromanomètre ouvert, la vérification pourra s'effectuer. Cette variation très faible de pression sera tirée de la différence de densité de l'air et du gaz carbonique. Un tube de verre étranglé vers le bas est relié par un caoutchouc à l'ampoule $\mathrm{A}$ du micromanomètre. Les déplacements de ce tube dans l'air n'ont aucun effet sur l'équilibre de la bulle, parce que le gaz intérieur compense la pression du gaz extérieur. Si on enfonce le tube dans une éprouvette remplie de gaz carbonique, la bulle glisse aussitôt, mais prend un équilibre. A ce moment, si l'on relève ou enfonce de 1 millimètre le tube de verre, la bulle glisse dans un sens ou dans l'autre, indiquant une diminution ou un accroissement de pression. Or le calcul montre que ce déplacement de 1 millimètre correspond à une variation de pression inférieure à $1 \mu$ d'eau ou à $\frac{1}{10}$ de barye.

Tout se passe comme si l'on enfonçait un tube dans un gaz de densité $0, \check{2} 29$. On voit donc que l'appareil peut mettre en évidence une variation de pression égale à celle qui existe dans l'air entre deux points distants verticalement de 0,0 millimètre. Il convient par suite pour la statique des gaz et pour répéter les expé- 
riences de Pascal devant une classe. Un tube de 1 litre de capacité rempli d'air à 30 centimètres au-dessus du micromanomètre, puis fermé, est apporté à la hauteur de ce dernier, par communication on constate dans ce tube une pression inférieure à celle du micromanomètre. La prise étant faite à 30 centimètres au-dessous, on constate au contraire une pression supérieure.

Le mouvement si facile de la bulle-index s'explique en remarquant que son enveloppe liquide glisse dans le même liquide constituant une gaine à l'intérieur du tube capillaire.

Cette bulle constitue cependant une bonne séparation entre les deux colonnes liquides. En effet il n'est pas nécessaire que le tube capillaire soit exactement horizontal pour que l'équilibre s'y produise; or, si la coupure n'était pas faite, la poussée du principe d'Archimède ferait glisser la bulle, le liquide pouvant passer d'un côté à l'autre. La vérification a été exécutée de la manière suivante : Deux ámpoules ont été réunies par un tube capillaire à section elliptique dont le grand axe est vertical. On remplit l'ensemble de tétrachlorure légèrement coloré, et l'on fait apparaître la bulleindex. On voit au-dessous d'elle un filet liquide coloré, car elle ne touche pas exactement le verre à cet endroit. Or il est extrêmement difficile de la maintenir en équilibre. La moindre inclinaison de l'appareil provoque le déplacement de celle-ci sur une longueur de plusieurs décimètres. Cela tient à ce que cette poussée d'Archimède peut être active, grâce à la communication de part et d'autre par le filet liquide.

L'expérience montre qu'il suffit de donner à cette bulle une longueur de 8 millimètres pour qu'elle fonctionne bien. Toutefois on devra éviter des variations très brusques et très notables de la pression.

La bulle se forme au moment du remplissage quand il y a peu de liquide ou bien encore après, il suffit de souffler de l'air sec dans le fond d'une des ampoules pour provoquer la formation de nombreuses bulles. On choisit entre elles. Il est encore possible de la créer par une inclinaison autour du tube capillaire servant d'axe horizontal. Les courbures du tube capillaire s'opposent à son départ.

Ce micromanomètre est très supérieur au manomètre de Kretz. Dans ce dernier, l'adhérence irrégulière des liquides à la paroi de verre provoque des déformations du ménisque de séparation rendant toute précision illusoire. 
Ce défaut est supprimé dans le micromanomètre. Partout le liquide est homogène; il n'y a pas de grippement. Le frottement rencontré par la bulle d'air est si faible qu'on n'aperçoit pas de déformation de ses ménisques même en les grossissant. Après fonctionnement de l'appareil dans un sens, un accroissement de pression de $1 \mu$ d'eau suffit pour faire glisser la bulle en sens inverse.

Aussi cet appareil peut-il servir à mettre en évidence de très faibles variations de pression.

Dans des recherches comportant des mesures, il faudra tenir compte des effets de capillarité aux cercles de raccordement dans les ampoules. Quand l'appareil vient d'être rempli, un déplacement de 120 millimètres de la bulle index ne provoque pas de changement de zéro. A ce moment les surfaces libres glissent sur la gaine du liquide adhérente aux ampoules. Pendant quelques minutes on constate un déplacement du zéro qui ne dépasse pas 1 millimètre. La bulle ne revient pas tout à fait à sa place primitive. Cela tient à ce que le bord des surfaces libres ne subit pas un déplacement aussi grand que le centre, car celle qui s'élève frotte contre le verre par adhérence du liquide et celle qui descend, pour la même raison, reste accrochée.

La bulle indique bien le zéro actuel; car si on la pousse légèrement vers l'ancien, elle revient d'elle-même dans sa situation d'équilibre.

On peut remarquer qu'au retour la bulle subit un déplacement presque égal à celui que lui avait donné l'accroissement de pression.

On est donc en droit de dire que les déformations des surfaces capillaires sont comparables et que l'erreur de capillarité est représentée par l'écart des zéros à très peu de chose près.

Si l'on néglige cette correction, on voit qu'elle entraine une erreur relative de $\frac{1}{120}$, ce qui correspond à une pression de $\frac{3}{4}$ de millimètre d'eau. Il sera préférable d'effectuer la correction.

L'appareil se prête bien à des comparaisons de faibles pressions presque égales; en effet les erreurs de capillarité et de calibrage étant très voisines, dans le quotient ces erreurs de même sens et de valeurs presque identiques se détruisent.

11 est évident que l'on devra éviter le plus possible l'évaporation. 


\section{APPLICATIONS.}

Cette sensibilité remarquable rend l'appareil applicable à un grand nombre de démonstrations et de mesures.

Voici un certain nombre d'entre elles:

$1^{\circ}$ Excès de pression intérieure dans les bulles liquides. - Pour les projections, on prend le modèle à tube capillaire assez court, tandis que pour les mesures on emploie le manomètre à ampoules cylindriques et à tube capillaire allongé.

Il est facile de montrer que la pression est supérieure dans la bulle à la pression atmosphérique du moment. En répétant avec le même liquide glycérique l'expérience précédente en diminuant le rayon, l'élève voit bien que l'accroissement de pression est d'autant plus grand que le rayon est plus faible.

On pourrait d'ailleurs étudier la pression dans les systèmes laminaires : nodoïdes, caténoïdes, onduloïdes.

La bulle de savon soufflée à l'extrémité d'un tube $\mathrm{T}$ est mise en relation avec l'une des ampoules, comme l'indique la fig. 3 .

On pent demander à des élèves de vérifier la loi de Laplace sur la bulle de savon; dans ce cas les ampoules sont élargies et le tube capillaire est allongé jusqu'à $\mathbf{4 0}$ centimètres environ. Le rayon de la bulle est mesuré au pied à coulisse. L'accroissement de pression $p$ étant égal à $4 \frac{\mathrm{A}}{\mathrm{R}}$, on a $: p \mathrm{R}=4 \mathrm{~A}$.

Il suffit de vérifier que le produit de la pression par le rayon est un nombre constant. Or la pression $p$ étant proportionnelle au déplacement de la bulle, il suffit d'établir que le produit de ce déplacement par le rayon correspondant $\mathrm{R}$ conduit à une constante.

On a d'ailleurs une mesure de la tension superficielle, si l'on déduit de l'appareil la valeur absolue de la pression $p$.

$2^{\circ}$ Mise en évidence de la pression électrostatique. - La fig. 4 montre la bulle de savon $\mathrm{S}$ soufflée à l'extrémité d'un tube d'ébonite $\mathrm{E}$ raccordé à un tube de verre $\mathrm{T}$. Un anneau métallique $\mathrm{M}$ terminant le tube d'ébonite est relié à la machine électrostatique disposée avec un trop-plein électrique. La bulle de savon $S$, fuyant facilement quand elle est électrisée, est retenue par un cercle de fil métallique disposé dans un plan vertical. Elle s'échappe quand même pour de hauts potentiels. 
Dans ce dernier cas, il est préférable d'utiliser le dispositif de M. Chassagny légèrement complété ( $f g$. こ) $)$.

La membrane élastique est surchargée d'un hémisphère de celluloïd très léger, fermé par une lamelle d'aluminium. L'aluminium repose par l'intermédiaire d'une rondelle sur la lamelle élastique. Le tout est bien métallisé (poudre d'aluminium) et peut être relié à une machine électrostatique. La boîte supportant la lamelle de caoutchouc est reliée par un tube isolant E à l'ampoule du micromanomètre.

$\mathrm{Au}$ moment de l'électrisation la pression électrostatique soulève l'hémisphère et la bulle-index se déplace. Entre les pôles de la machine, munie du trop-plein, on augmente la distance : le déplacement de la bulle grandit en même temps. Vient-on à faire varier brusquement le potentiel par éclatement d'étincelles, la bulle traduit chaque variation.

$3^{\circ}$ Mesure d'un volume très petit. - Deux cylindres C et D dont le rayon est d'un centimètre environ sont reliés par le tube capillaire. Le tout contient du liquide; la bulle-index est en place dans le tube capillaire gradué en parties d'égale longueur $(f g .6)$.

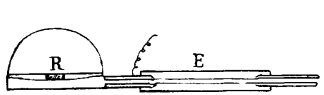

FIG. ว.

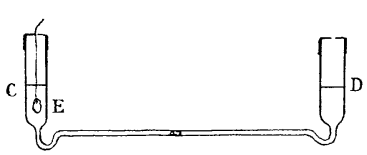

FIG. 6 .

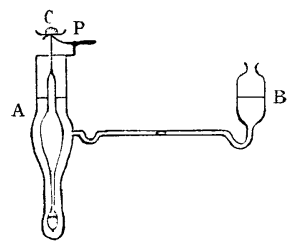

FIG. 7.

En plongeant le corps dont on veut le volume, la bulle se déplace d'une quantité qui est proportionnelle au volume du corps E. Théoriquement le volume décrit par la bulle est la moitié de celui du corps.

On pourrait chercher ce volume V. Mais il est plus sûr d'opérer de la manière suivante pour faire disparaître presque totalement les erreurs de capillarité et même de calibrage. Le corps E est remplacé par un étalon ayant à peu près le même volume $V^{\prime}$. On écrit que les volumes sont entre eux comme les déplacements de la bulle. Comme on opère dans des conditions analogues, les erreurs absolues sont de même sens.

Dans le quotient les erreurs relatives se soustraient; commes elles 
sont presque égales, l'erreur relative sur la mesure est très faible.

Deux fragments d'un même laiton, ayant les masses respectives 6 et 5 grammes, ont aussi les volumes respeciifs 6 et ๖. En supposant connu le plus petit, évalué en unité arbitraire, soit $ّ$, on trouve ainsi pour le premier 6,002 .

$4^{\circ}$ Mesure d'une masse pouvant s'élever jusqu'à quelques grammes.

- L'ampoule A est allongée vers le bas. On y introduit un flotteur lesté par une bonne masse de mercure et portant un plateau P. Il est facile de voir que le déplacement de la bulle est proportionnel à la masse du corps $\mathrm{C}$ posé sur le plateau ( $f g$. 7). On pourrait donc se contenter de chercher le déplacement qui convient à 1 gramme, une règle de trois permettrait de calculer la masse du corps en expérience après lecture du déplacement correspondant.

Comme on l'a vu dans la recherche d'un volume, on aura une précision meilleure en lisant le déplacement donné par une masse marquée de grandeur voisine et écrivant le rapport entre les masses et les déplacements.

Un modèle construit de cette façon donnait la masse à 2 milligrammes près.

L'appareil est rapide; il est apériodique et l'équilibre est obtenu en moins de deux minutes.

Il peut avantageusement remplacer le trébuchet des laboratoires.

On peut diminuer la longueur de la course de l'index en plaçant sur le flotteur des plateaux ou des masses étalonnées, qu'on enlèverait au moment où l'on y place le corps dont on cherche la masse.

Il suffirait d'ajouter à la masse connue du plateau ou de la masse étalonnée le supplément correspondant au déplacement de la bulle.

Un levier permettant de soutenir le plateau rend les opérations faciles.

$5^{\circ}$ Mise en évidence de faibles quantités de chaleur ou de faibles élévations de température. - La chaleur apportée au gaz de l'ampoule $\mathrm{B}$ fermée accroît la pression de celui-ci; d'où un déplacement de la bulle-index ( $f g .8$ ).

En mastiquant un tube d'aluminium mince, on met en évidence la chaleur créée par le travail en frottant le tube à l'intérieur; une seule rotation d'un frotteur suffit pour déplacer la bulle d'une manière visible.

En montant le même tube dans l'ampoule A, on a un appareil dif- 
férentiel pouvant permettre de démontrer l'inégalité des chaleurs spécifiques.

Afin de faciliter la démonstration, on laisse A et B en communication avec l'air par une ouverture latérale que l'on ferme au moment d'apporter dans le tube les deux corps de masses égales.

La fig. 9 montre un appareil très commode pour mettre en évidence le phénomène de Peltier.

Une des soudures de la pile thermo-électrique est enfermée dans l'ampoule A, et l'autre, dans l'ampoule B.

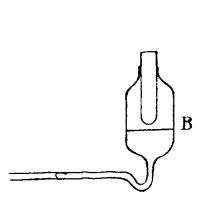

FIG. 8 .

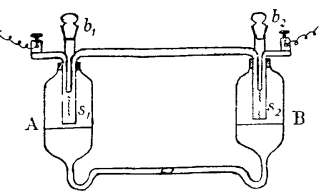

FIG. 9.

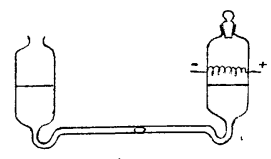

FIG. 10.

On fait passer le courant, l'index glisse dans un sens; on renverse le courant et l'index se meut en sens contraire. Des tubes à bouchons $b_{1}$ et $b_{2}$ rendent l'opération facile.

La chaleur dégagée dans un fil se montre de la même façon (fig. 10).

$6^{\circ}$ Mesure du pouvoir inducteur spécifique. - Le flotteur du micromètre-balance porte un plateau circulaire $\mathrm{C}$ entouré d'un anneau de garde G, le tout étant relié au sol (fig. 11).

$\mathrm{Au}$-dessous de $\mathrm{G}$ se trouve un plateau fixe $\mathrm{P}$, au-dessus un plateau mobile $Q$, dont la distance au plan del'anneau de garde peut se mesurer.

Les plateaux $\mathrm{P}$ et $\mathrm{Q}$ sont reliés métalliquement entre eux et avec l'un des pôles d'une bobine de Ruhmkorff dont le second pôle est au sol.

L'appareil étant réglé, on écarte $Q$ de telle façon que la décharge de la bobine n'agisse pas sur C.

La position de $Q$ étant repérée, on place entre $C$ et $Q$ une lame de la matière isolante reposant sur trois cales disposées sur l'anneau de garde.

C est attiré vers le haut; on relève $Q$ d'une hauteur $d$, de telle sorte que l'équilibre soit rétabli, c'est-à-dire jusqu'à ce que la bulle-index ait repris sa position d'équilibre. On sait qu'en appelant $\mathrm{K}$ le pou- 
voir inducteur spécifique, e l'épaisseur du diélectrique, on a la relation $\mathrm{K}=\frac{e}{e-d} \cdot$ L'équilibre est stable.

Pour effectuer le réglage, on ajoute ou on retranche du liquide, ou encore on surcharge un petit plateau supplémentaire R. L'appareil est apériodique.

$7^{\circ}$ Mesure d'une différence de potentiel en valeur absolue. L'appareil rappelle celui de MM. Abraham et Lemoine. Il sert également pour des potentiels assez élevés.

Le plateau $\mathrm{C}$ mobile est fixé au flotteur du micromanomètre balance et entouré d'un anneau de garde $G$; le tout est relié au sol (fig. 12).

Le plateau Q, porté au poténtiel à mesurer, est déplaçableà volonté. Sa distance au plateau de l'anneau de garde peut s'évaluer.

On règle l'appareil de telle sorte que le plateau $\mathrm{C}$ coïncide avec le plan de l'anneau de garde, soit en faisant varier la quantité de liquide soit en surchargeant le petit plateau $R$.

On dispose ensuite sur $R$ une masse étalon convenable et on cherche la distance du plateau $\mathrm{Q}$, chargé à un potentiel invariable, qui correspond à l'équilibre. La bulle doit rester au point qui correspond au réglage.

L'appareil est apériodique et utilise un équilibre stable.

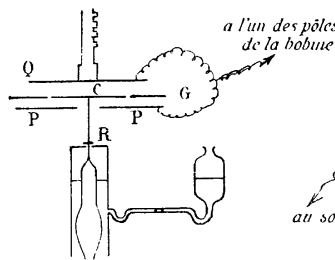

FIG. 11.

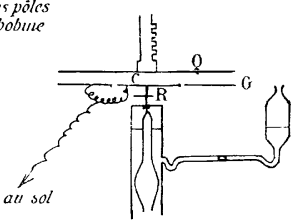

FIG. 12.

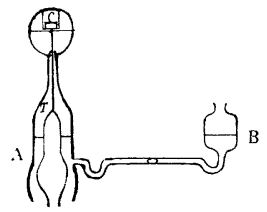

FIG. 13.

Un deuxième appareil n'est autre que celui de M. Lippmann (fig. 13).

L'hémisphère supérieur est fixé au flotteur du micromanomètrebalance; l'inférieur est supporté par un verre ayant à peu près la forme conique et prolongeant l'ampoule A. Dans l'axe de ce verre conique passe la tige de verre $\mathrm{T}$ solidaire de l'hémisphère supérieur.

J. de Phys., כّ॰ série, t. III. (Août 1913.) 
Afin de pouvoir effectuer des mesures, on alourdit plus ou moins l'hémisphère supérieur.

On a, à cet effet, réservé en dedans une cavité dans laquelle on place une masse étalonnée.

La cavité se ferme par une calotte sphérique doublée de fer. Un aimant permet de l'ouvrir à volonté. L'équilibre étant rétabli quand le potentiel est nul, ce qui se réalise en ajoutant ou retranchant du liquide, on met dans la cavité une masse $m$ connue. On cherche à faire varier le potentiel (machine à trop plein) de telle sorte que l'hémisphère supérieur soit tiré vers le haut par la pression électrostatique. Le potentiel est donné par la relation $m g=\frac{\mathrm{V}^{2}}{8} \cdot \mathrm{En}$ augmentant la masse $m$, on peut passer à la mesure d'un potentiel plus élevé.

L'équilibre est encore stable.

$8^{\circ}$ Mesure de la densité d'un gaz par rapport à l'air. - Le micromanomètre a ses ampoules légèrement modifiées. Elles sont fermées par une plaque plane de verre portant: $1^{\circ}$ une ouverture avec bouchon rodé $b$ et un tube $t$, recourbé horizontalement vers le bas. Le tube capillaire a environ 30 centimètres de longueur. L'appareil est complété par un tube de verre ayant environ 2 centimètres de diamètre et dont la hauteur est de $1^{\mathrm{m}}, 25$ environ $(f \mathrm{~g} g .14)$. Ce tube porte une ouverture en $O$. On peut le relier par $c$ au générateur de gaz pur et sec.

Soit à mesurer la densité du gaz carbonique par rapport à l'air.

On tourne l'orifice $O$ vers le haut, la partie rétrécie e étant au niveau de la surface libre du liquide. La hauteur d'air restant dans l'ampoule $B$ est très faible.

On repère la position de la bulle-index sur le tube gradué. Puis on remplit le tube de gaz carbonique la communication en $e$ étant coupée. On ouvre ensuite en $e$. La bulle se trouve refoulée d'une longueur $l$. Fn désignant par $a$ la masse du centimètre cube d'air dans les conditions de l'expérience, par $\mathrm{H}$ la hauteur du tube entre $O$ et $e ; x$ la densité du gaz carbonique par rapport à l'air; $s$ et $\mathrm{S}$ les sections droites du tube capillaire et des ampoules et enfin par $d$ la densité du liquide utilisé dans l'appareil, on a la relation :

$$
2 l \frac{s}{\mathrm{~S}} d=\mathrm{H} a(x-1) \text {. }
$$

Elle permettrait de déterminer $x$; mais l'erreur relative serait 
assez notable à cause des nombreuses grandeurs qui entrent dans l’équation.

Recommençons l'expérience avec l'hydrogène, le tube étant cette fois vers le bas comme sur la fig. 14. Soit $l^{\prime}$ le déplacement de la bulle-index ; $\delta$ la densité de l'hydrogène par rapport à l'air. On a encore :

$$
2 l^{\prime} \frac{s}{\mathrm{~S}}=\mathrm{H} a(1-\delta) .
$$

En divisant les équations (1) et (2) membre à membre, on obtient :

$$
\frac{l}{l^{\prime}}=\frac{x-1}{1-\delta}=\frac{x-1}{0,9305}
$$

D'où l'on tire la valeur de $x$.

On a trouvé ainsi avec un tube non calibré et des gaz secs, mais non rigoureusement purs, des résultats aussi exacts qu'on pouvait l'espérer.

Le procédé est applicable à des gaz industriels ; il n'exige que quelques minutes. On peut l'utiliser pour les gaz carbonique, sulfureux, le gaz de l'éclairage, etc. Il faut seulement éviter le contact du gaz et du liquide, quand ce gaz est soluble dans Je liquide. Comme ce dernier est saturé d'air, il vaut mieux empêcher le gaz d'arriver dans l'ampoule en plaçant un peu d'ouate dans un renflement situé entre $e$ et l'ampoule.

Pour des mesures précises, on devrait maintenir les deux gaz à la même température; ou du moins tenir compte de leur différence de température.

$9^{\circ}$ Mesure de la densité d'un solide. - On a vu que le volume d'un solide pouvait se mesurer par l'appareil décrit ( $f g .6$ ).

D'autre part le micromanomètre-balance fournit l'a masse du corps. On peut ainsi en quelques minutes trouver la densité d'un solide à $\frac{1}{200}$ près.

$10^{\circ}$ Débit d'un tuyau parcouru par un courant gazeux. - On sait que pour un tuyau cylindrique le débit est proportionnel à la vitesse du gaz. D'autre part l'expérience apprend que la différence de pression pour deux points du tuyau est proportionnelle à la vitesse. Il suffit donc d'avoir une indication $l$ proportionnelle à cette différence de pression pour représenter le débit $\mathrm{V}$ par $\mathrm{K} l$. 
664 HENRY. - MICROMANOMĖTRE ET DISPOSITIFS DIVERS

Or le déplacement de la bulle-index quand les ampoules sont reliées à deux points du tuyau est proportionnel à la différence de pression.

D'où :

$$
\mathbf{V}=\mathbf{k} l \text {. }
$$

Si l'on change la vitesse de passage, le reste étant identique, on pourra, en estimant la nouvelle valeur $l^{\prime}$ du déplacement, obtenir $\mathrm{V}^{\prime}=\mathrm{K} l^{\prime}$. En remplaçant $\mathrm{K}$ par sa valeur, on aura :

$$
\mathrm{V}^{\prime}=\frac{\mathrm{V}}{l} \cdot l^{\prime}
$$

$12^{\circ}$ Pression d'une onde sonore sur le plan où elle se réfléchit. La fig. 15 indique le dispositif adopté.

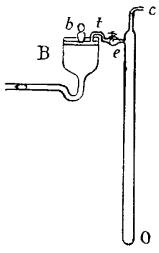

FIG. 14.

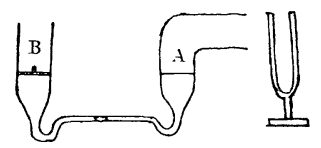

FIG. 15.

L'ampoule B est reliée par un tube de caoutchouc avec une chambre où l'air est calme. L'onde sonore émanée du diapason entretenu électriquement frappe la surface libre du tétrachlorure en A. Une dénivellation se produit, traduite par la bulle-index. La pression exercée par l'onde dépend de l'amplitude et de l'état de la surface réfléchissante. En réalité pour éviter l'évaporation du tétrachlorure de carbone, on a posé sur lui un flotteur analogue à un couvercle de boîte retourné et très léger en mica très mince dont la lamelle adhère au tétrachlorure. En mettant dans ce flotteur des corps mous on fait varier la pression de l'onde pour une même amplitude. 